\title{
A new finite-field mass reconstruction algorithm
}

\author{
S. Seitz ${ }^{1}$ and P. Schneider ${ }^{2}$ \\ 1 Universitätssternwarte München, Scheinerstr. 1, 81679 München, Germany \\ 2 Institut für Astropysik und Extraterrestische Forschung, Auf dem Hügel 71, 53121 Bonn, Germany
}

Received 10 February 1998 / Accepted 30 March 2001

\begin{abstract}
A new method for the reconstruction of the projected mass distribution of clusters of galaxies from the image distortion of background galaxies is discussed. This method is essentially equivalent to the one we developed previously, i.e., the noise-filtering method, but has several practical advantages: (1) it is much easier to implement; (2) it can be easily applied to wide-field images, since the constraints on the number of gridpoints are much weaker than for the previous method, and (3) it can be easily generalized to more complicated field geometries, such as that of the Wide Field Planetary Camera 2 (WFPC2) onboard HST. We have tested the performance of our new inversion method (for which a FORTRAN-77 implementation is available from the authors) using simulated data, demonstrating that it fares very favourably.
\end{abstract}

Key words. cosmology: theory - methods: numerical

\section{Introduction}

The distortion of the images of background galaxies (Tyson et al. 1990) by the tidal gravitational field of clusters of galaxies can be used to obtain a parameter-free reconstruction of the surface mass density of the cluster (Kaiser \& Squires 1993). Several modifications of the original reconstruction method were proposed, e.g., to account for distortions which are not weak (Seitz \& Schneider 1995; Kaiser 1995), to allow an unbiased mass reconstruction on a finite field (Schneider 1995; Kaiser et al., astro-ph/9411029; Bartelmann 1995; Bartelmann et al. 1996; Seitz \& Schneider 1996, hereafter Paper I; Squires \& Kaiser 1996), and to account for a broad redshift distribution of the background galaxies (Seitz \& Schneider 1997). In this paper, we shall reconsider the second of the above mentioned effects, namely mass reconstructions from data on a finite field. In Paper I we have derived a direct mass inversion method which is singled out of the infinitely-many unbiased reconstructions by identifying a component of the noise (which is due to the intrinsic ellipticity distribution of the sources, the discreteness of galaxy images, and observational effects) as such and filtering it out. This noise-filter reconstruction has fared very well in numerical simulations carried out to compare various finite-field inversions (Paper I; Squires \& Kaiser 1996). The distortion of the images of background galax-

Send offprint requests to: S. Seitz,

e-mail: stella@usm.uni-muenchen.de ies (Tyson et al. 1990) by the tidal gravitational field of clusters of galaxies can be used to obtain a parameter-free reconstruction of the surface mass density of the cluster (Kaiser \& Squires 1993). Several modifications of the original reconstruction method were proposed, e.g., to account for distortions which are not weak (Seitz \& Schneider 1995; Kaiser 1995), to allow an unbiased mass reconstruction on a finite field (Schneider 1995; Kaiser et al., astro-ph/9411029; Bartelmann 1995; Bartelmann et al. 1996; Seitz \& Schneider 1996, hereafter Paper I; Squires \& Kaiser 1996), and to account for a broad redshift distribution of the background galaxies (Seitz \& Schneider 1997). In this paper, we shall reconsider the second of the above mentioned effects, namely mass reconstructions from data on a finite field. In Paper I we have derived a direct mass inversion method which is singled out of the infinitely-many unbiased reconstructions by identifying a component of the noise (which is due to the intrinsic ellipticity distribution of the sources, the discreteness of galaxy images, and observational effects) as such and filtering it out. This noise-filter reconstruction has fared very well in numerical simulations carried out to compare various finite-field inversions (Paper I; Squires \& Kaiser 1996).

Here, we shall present a slightly revised version of the noise-filter inversion method, which removes some of the technical drawbacks of the original formulation. In particular, our new method can be applied to arbitrarily-shaped data fields (which is of great interest given the geometry of the WF chips of the WFPC2 on-board HST) and can 
be used with better resolution than the previous formulation. In addition, the numerical encoding of the new version is substantially easier and requires much less memory. We shall formulate the inversion problem and its solution in Sect. 2, and present some practical issues in Sect. 3. Numerical tests of this method in comparison to other reconstruction methods are presented in Sect. 4, and we summarize in Sect. 5 our findings. One application of our new method has been presented in the mass reconstruction of the cluster MS1358+62 by Hoekstra et al. (1998).

Just before finalizing this manuscript, Lombardi \& Bertin (1999) submitted a paper to the astro-ph preprint server. Two results of that paper are particularly relevant for the present discussion: They have shown that of all (direct) finite-field mass reconstructions, those with vanishing curl in the kernel $\boldsymbol{H}$ - see Eq. (6) below - have the smallest rms error; requiring that noise-free data yield an exact mass reconstruction, they rederived the inversion method of Paper I. Second, they have independently derived our new inversion method, Eq. (7) below, from a variational principle.

\section{Noise-filtered finite-field mass inversion}

We shall assume for simplicity that all source galaxies can be described as being at the same redshift; this is not a necessary assumption (see Seitz \& Schneider 1997), but simplifies the following treatment considerably. Then, let the mass distribution of the cluster be described by the dimensionless surface mass density $\kappa(\boldsymbol{\theta})$, and the corresponding deflection potential be denoted by $\psi(\boldsymbol{\theta})$, such that the two-dimensional Poisson equation $\nabla^{2} \psi=2 \kappa$ is satisfied. The two components of the complex shear $\gamma=\gamma_{1}+\mathrm{i} \gamma_{2}$ are given in terms of the deflection potential by

$\gamma_{1}=\frac{1}{2}\left(\psi_{, 11}-\psi_{, 22}\right), \quad \gamma_{2}=\psi_{, 12}$,

where indices separated by a comma denote partial derivatives $^{1}$. The complex reduced shear

$g(\boldsymbol{\theta})=\frac{\gamma(\boldsymbol{\theta})}{1-\kappa(\boldsymbol{\theta})}$

is the expectation value of the observed image ellipticities $\epsilon$, so that the observed image ellipticities provide an unbiased estimate of the local value of $g$, as long as the cluster is non-critical. We shall make this assumption here, although it also can be dropped (see Seitz \& Schneider 1997). As pointed out by Schneider \& Seitz (1995), the mass-sheet degeneracy (Gorenstein et al. 1988) allows one to determine $(1-\kappa)$ only up to a multiplicative constant, if no magnification information is used (Broadhurst et al. 1995; Bartelmann \& Narayan 1995). Defining

$K(\boldsymbol{\theta}):=\ln [1-\kappa(\boldsymbol{\theta})]$,

1 Note that we have changed the sign convention compared to Paper I. then $K$ can only be determined up to an additive constant. Kaiser (1995) derived a relation between the gradient of $K$ and combinations of first derivatives of $g$,

$\nabla K=$

$$
\frac{-1}{1-|g|^{2}}\left(\begin{array}{cc}
1-g_{1} & -g_{2} \\
-g_{2} & 1+g_{1}
\end{array}\right)\left(\begin{array}{c}
g_{1,1}+g_{2,2} \\
g_{2,1}-g_{1,2}
\end{array}\right) \equiv \boldsymbol{u}(\boldsymbol{\theta}) \text {. }
$$

The right-hand-side of this equation can be considered as an observable, obtained from local averages of image ellipticities and by finite differencing the resulting field $g$.

Equation (4) can be solved (up to an additive constant) by line integration, and several schemes for this have been proposed (Schneider 1995; Kaiser et al. 1995; Bartelmann 1995; Squires \& Kaiser 1996). The reason why different schemes yield different results can be seen by noting that the vector field $\boldsymbol{u}$ comes from (noisy) observational estimates, and thus will in general not be a gradient field. Therefore, the equation $\nabla K=\boldsymbol{u}$ has no solution in general, since $\boldsymbol{u}$ has a rotational component due to observational noise. On the other hand, if $\boldsymbol{u}$ is a gradient field, then all line integration schemes are equivalent.

In Paper I, we split the vector field into a gradient part and a rotational part,

$$
\boldsymbol{u}(\boldsymbol{\theta})=\nabla \tilde{K}(\boldsymbol{\theta})+\operatorname{rot} s(\boldsymbol{\theta}) \equiv \nabla \tilde{K}(\boldsymbol{\theta})+\left(\begin{array}{c}
\partial s / \partial \theta_{2} \\
-\partial s / \partial \theta_{1}
\end{array}\right),
$$

where $s(\boldsymbol{\theta})$ is a scalar field. This decomposition is not unique. However, since the rotational component is due solely to noise, we can specify the decomposition uniquely by requiring that the mean of rot $s$ over the finite data field $\mathcal{U}$ vanishes, and that $\operatorname{rot} s$ vanishes if $\boldsymbol{u}$ is a gradient field. These two conditions are satisfied if we set $s=$ const. on the boundary $\partial \mathcal{U}$ of the data field $\mathcal{U}$. Then, identifying $\nabla \tilde{K}$ with $\nabla K$, the solution of (4) with the rotational component removed from $\boldsymbol{u}$ becomes

$$
K(\boldsymbol{\theta})-\bar{K}=\int_{\mathcal{U}} \mathrm{d}^{2} \theta^{\prime} \boldsymbol{H}\left(\boldsymbol{\theta}^{\prime}, \boldsymbol{\theta}\right) \cdot \boldsymbol{u}\left(\boldsymbol{\theta}^{\prime}\right)
$$

where $\boldsymbol{H}\left(\boldsymbol{\theta}^{\prime}, \boldsymbol{\theta}\right)$ is a vector field which can be obtained from the Greens function of a Laplace equation with Neumann boundary conditions. In Paper I we have derived this equation and presented explicit solutions for the cases that $\mathcal{U}$ is a circle or a rectangle; in these cases, the Greens function can be obtained analytically using geometrical methods.

Whereas the method of Paper I passed all numerical tests, it has a few features which are unwanted: (1) If the geometry deviates from that of a circle or a rectangle, the Greens function can no longer be obtained analytically. However, a numerical determination of the Greens function is impractical owing to its singularity. For this reason, the mass reconstruction of the cluster $\mathrm{Cl} 0939+4713$ from WFPC2 data (Seitz et al. 1996) was carried out by splitting the field into two rectangles and combine them appropriately in the overlap region. This is certainly not the optimal method, since each of the two individual reconstructions made no use of the shear information outside 
the respective rectangle. (2) If a quadratic field is covered by an $N \times N$ grid of $\boldsymbol{\theta}$ and $\boldsymbol{\theta}^{\prime}$ values, the necessary memory for storing $\boldsymbol{H}$ consists of $2 N^{4}$ real numbers. Hence, if one increases $N$ beyond $\sim 50$, the memory requirement quickly approaches the capacity of commonly used workstations. However, due to the singularity of the Greens function, one likes to have small grid spacings to obtain an accurate numerical estimate of the integral (6) - see Squires \& Kaiser (1996) for comments on this point. (3) Although the solution for $\boldsymbol{H}$ was given explicitly in Paper I, it is complicated and not easily encoded (though quickly evaluated). In order for the noise-filtering method to become a standard and readily available tool, one would like to have an easier method to solve for $K$.

These three points can be avoided in the following simple manner: Taking the divergence of (5) leads to

$\nabla^{2} K=\nabla \cdot \boldsymbol{u}$

Since $s=$ const. on the boundary, rot $s$ is perpendicular to the normal vector $\boldsymbol{n}$ at the boundary of $\mathcal{U}$, so that

$\boldsymbol{n} \cdot \nabla K=\boldsymbol{n} \cdot \boldsymbol{u}$ on $\partial \mathcal{U}$

Hence, $K$ can be obtained as the solution of the Neumann problem given by $(7 \mathrm{a}, \mathrm{b})$. There are efficient and quick methods for a numerical solution of this problem; we have employed a relaxation method with successive overrelaxation (see Press et al. 1992, p. 857). Choosing the overrelaxation parameter as in Eq. (19.5.21) of Press et al. (1992), a stable solution was found after about $20 N$ iterations on an $N \times N$ grid in $\boldsymbol{\theta}$.

The previously mentioned drawbacks of the method presented in Paper I are now avoided. The Neumann problem (7) can be solved for any geometry; for example, for the WF-part of the WFPC2 one merely needs to formulate the boundary condition (7b) at 6 sides, instead of 4 for a rectangle. The memory requirement is reduced to a few times $N^{2}$ real numbers, so that $N$ can easily be of order a few hundred. In fact, for $N=200$, the solution of (7) takes about 2 min on an IBM risc 6000 processor. And finally, the numerical code for solving (7) shrinks tremendously compared to that needed to evaluate $\boldsymbol{H}$.

\section{Practical implementation}

In order to obtain a mass reconstruction from galaxy ellipticities, the following three steps are needed:

(1) The galaxy ellipticities are spatially smoothed to obtain an unbiased estimate of the local reduced shear. If $\epsilon_{i}$ is the complex ellipticity of the $i$-th galaxy at position $\boldsymbol{\theta}_{i}$, and $\Delta \theta$ is a smoothing scale, we calculate $g$ at a position $\boldsymbol{\theta}$ as

$$
g(\boldsymbol{\theta})=\frac{\sum_{i=1}^{N_{\mathrm{g}}} w\left(\left|\boldsymbol{\theta}-\boldsymbol{\theta}_{i}\right|\right) \epsilon_{i}}{\sum_{i=1}^{N_{\mathrm{g}}} w\left(\left|\boldsymbol{\theta}-\boldsymbol{\theta}_{i}\right|\right)},
$$

where the weight function $w$ is chosen to be

$$
w(x)=\exp \left(-\frac{x^{2}}{\Delta \theta^{2}}\right)-\exp (-q)
$$

for $x \leq \sqrt{q} \Delta \theta$, and zero otherwise. This choice makes $w$ nearly Gaussian and continuous at $x=\sqrt{q} \Delta \theta$, which can be an essential aspect when the derivatives of the components of $g$ need to be evaluated with finite differencing and $q$ is small. In the following we will choose $q=9$, where (9) becomes almost equal to the case where the correction term is omitted. With (8), the reduced shear $g$ can be calculated on a regular grid in $\boldsymbol{\theta}$.

(2) The vector field $\boldsymbol{u}$ is obtained from $g$ using (4). Finite differencing is employed, with one-sided secondorder differentiation rules taken at the boundary $\partial \mathcal{U}$. A further differentiation then yields $\nabla \cdot \boldsymbol{u}$.

(3) The Neumann problem (7) is then solved, using the method described above ${ }^{2}$.

\section{Tests and simulations}

One might wonder whether the mass reconstruction obtained with the method described above yields smooth mass profiles. Our method requires differentiation of (noisy) data, so it might be suspected that the resulting mass distribution will be quite noisy compared to the results of some of the other finite-field inversion methods. These issues have been discussed in some detail by Squires \& Kaiser (1996); whereas it is not a priori evident that these numerical differentiations are unharmful to the resulting mass reconstruction, the numerical simulations these authors have carried have shown, in agreement with Paper I, that the noise-filter inversion as presented in Paper I yields the least noisy mass estimates of all unbiased direct finite-field mass reconstructions that they have tested ${ }^{3}$. Since the method proposed here involves a further differentiation of the data, we have to check whether the noise level of the reconstruction is not increased by that. For this purpose, we have carried out two sets of simulations. In both cases, galaxies were distributed randomly on the data field $\mathcal{U}$, with a density of 50 galaxies per square arcminute and an intrinsic ellipticity distribution which is assumed to be a Gaussian of width $\rho=0.2$ (see Paper I). In the first set of simulations, a mass distribution for the cluster corresponding to the lens model B in Paper I was assumed (see Fig. 1 of Paper I, upper right panel), and the "observed" ellipticities were calculated from the intrinsic ellipticities and the local value of the reduced shear caused by the lens covering a field of $7^{\prime} .5 \times 7^{\prime} .5$. In the second set of simulations,

\footnotetext{
${ }^{2}$ A Fortran 77 code of these three steps is available from the authors by request, both for a rectangular data field, and the WFPC2 geometry.

3 Inverse methods, such as the maximum likelihood method (Bartelmann et al. 1996; Seitz et al. 1998) or the maximum probability method (Squires \& Kaiser 1996) can yield slightly more accurate mass profiles.
} 


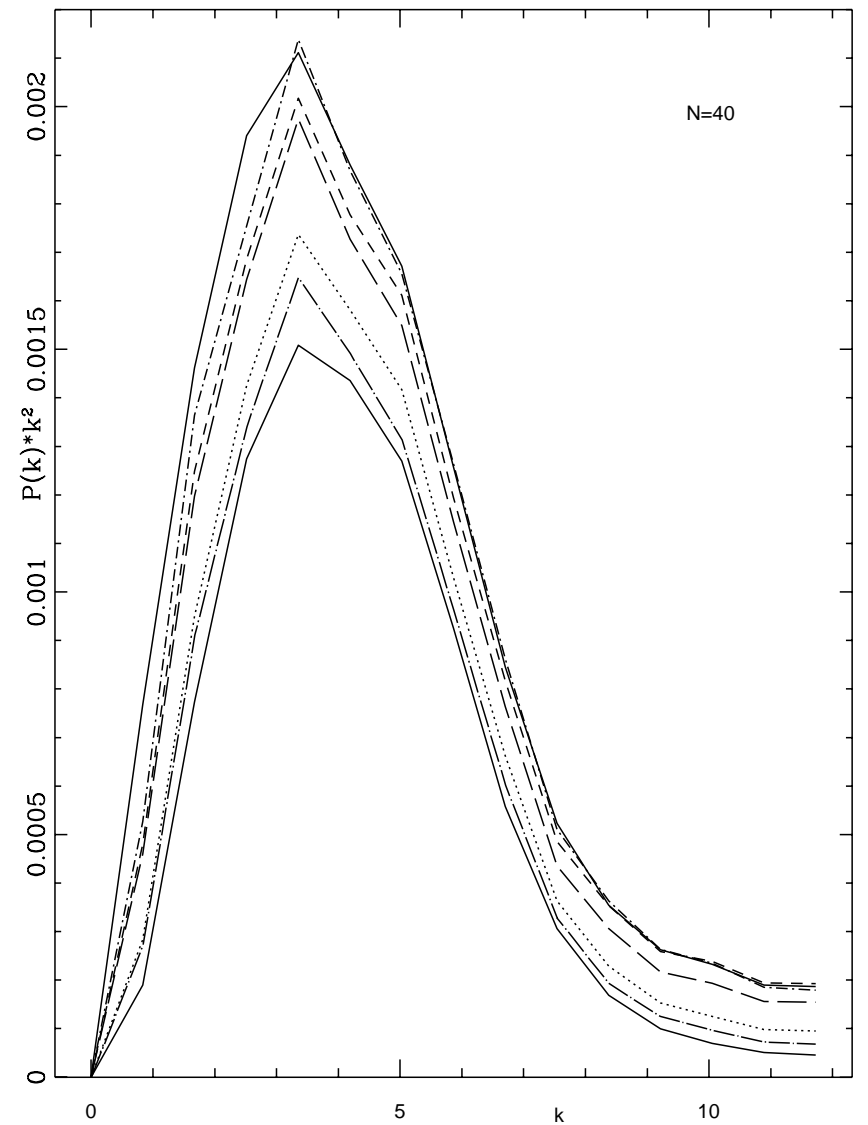

Fig. 1. The power spectra of various mass inversion methods, as explained in the main text.

no lens was assumed; owing to the intrinsic ellipticity of the sources, the reduced shear as calculated from the "observed" ellipticity does not vanish identically, and so the reconstructed mass profile will be different from zero (this is the kind of simulations carried out in Squires \& Kaiser 1996). The smoothing length was set to $\Delta \theta=0$ '.35.

Reconstructions for the case with a lens were performed using the following methods: the original Kaiser \& Squires (1993) reconstruction, generalized to account for non-linear effects as described in Paper I; a finite-field reconstruction based on line integration (Schneider 1995); the noise-filtering method (NF) as described in Paper I; and the new noise-filtering method as presented here. The reconstructions were analyzed by Fourier-decomposition of their difference from the input mass distribution (or, more precisely, the input field of $K$ ). We used several values of the number of gridpoints per dimension. The noise filtering inversion developed here was implented in two versions (NF1, NF2); in the first case, (7a\&b) was solved on the same grid as for the other inversion techniques. In the second case, the solution for $K$ was obtained on a grid two times as dense and $K$ was estimated on the sparser grid afterwards.

It turns out that reconstructions with the new technique developed here (NF1) are always smoother than any of the other methods considered here. This is because $\nabla \cdot \boldsymbol{u}$ is used instead of $\boldsymbol{u}$ itself. The operation $\nabla \cdot \boldsymbol{u}$ effectively yields a loss of signal and noise on length scales of two grid points. To obtain reconstructions with the same resolution we thus double the number of gridpoints in NF2, calculate $\boldsymbol{u}$ and its divergence and $K$ on the dense grid. Finally $K$ is calculated on the sparser grid by averaging over 4 gridpoints. The power spectra of these NF2 reconstructions are always very similar to the original NF-reconstruction. Those of the NF1 reconstructions are more similar to reconstructions on a sparser grid, where the high frequency power is reduced due to the loss of degrees of freedom. Hence, the recovery of the signal and the sensitivity to the noise in the NF2 and NF-method are identical, and therefore the comparisons with the other methods given in Paper I apply. To compare our results with those of Squires \& Kaiser (1996) we also consider the case with no shear and surface density in the data field (i.e. the "noiseonly-case"). This approach investigates the quality of the reconstruction (the quality of the "no-mass-detection") for the case that there is no mass in the field at all, whereas we have investigated before how good a two-dimensional mass distribution can be recovered. Given that a method which involves a lot of smoothing will always fare better in the no-lens case than one which spatially resolves noise, it is clear that the no-lens comparison is not the relevant test [the "best" inversion in that case is obtained by setting $\boldsymbol{H} \equiv \mathbf{0}$ in $(6) !]$

We found again that the noise properties of NF and NF2 reconstructions are (almost) identical, whereas that of NF1 is different for reasons already discussed. For a dense grid $(N=80)$ all noise filtering methods become more and more equal, and the short wavelength behavior approaches that of KS (solid line). In any case, the KS method is by far the "best" as long as there is no mass in the field. As we already pointed out in Paper I, this is because more (and exact) information is used, namely that the shear is (set equal to) zero outside the data field. The fact that the noise of the KS inversion in Fig. 6 of Squires \& Kaiser (1996) is slightly larger than that of the NF inversion at small wavelengths is due to the fact that in their implementation of the KS algorithm, the shear field was not obtained by smoothing the galaxy ellipticities, but the inversion was performed by straight summation, which leads to shot noise (Seitz \& Schneider 1995).

Squires \& Kaiser (1996) suspected that the increase of noise of the finite field inversion comes from the fact that they are more sensitive to noise at the boundary of the data field. This point is clarified in Fig. 1. The upper and lower solid curves denote the power spectra for the $\mathrm{NF}$ and $\mathrm{KS}$ method on a $40 \times 40$ grid. The underlying galaxy distribution and thus shear field for each of the individual reconstructions is by construction absolutely the same for the NF and KS-case. We then embed the true data field $\mathcal{U}$ in a two times as large field and distribute additional galaxies with the same density and ellipticity distribution in the outer region. The galaxies inside $\mathcal{U}$ are unchanged. The shear field is calculated in the large field and KS-reconstructions are obtained in the same region. We cut out the surface mass density in the original field $\mathcal{U}$ 
and calculate the power spectrum of the reconstruction error in the same way as for the other mass reconstructions within $\mathcal{U}$. We point out that in this case the shear field within $\mathcal{U}$ is not the same as in the above case because now galaxies outside the field contribute to the estimate of the shear field within $\mathcal{U}$. This makes the shear field statistically smoother inside $\mathcal{U}$. But as can be seen in Fig. 1 (longdashed-dotted curve) the reconstruction error within $\mathcal{U}$ is larger than for KS-reconstructions of the small field (solid line) - because the data outside $\mathcal{U}$ are no longer "ideal assumptions" ( $\gamma \equiv 0)$ but noisy measurements affected by the intrinsic ellipticity distribution of the galaxies. We then perform reconstructions on the large field where the shear field is obtained in the same way as before, but values on gridpoints within $\mathcal{U}$ are substituted by the estimate obtained in the small field only. Thus the $g$ field and its noise properties within $\mathcal{U}$ are now identical to that of the $\mathrm{KS}$ and NF reconstructions of the small field. At the same time the transition to the shear field outside $\mathcal{U}$ becomes less continuous which mimics an artifical increase of noise at the boundary of $\mathcal{U}$. The power spectrum obtained from KS-reconstructions of that $g$-field (dotted curve) is higher than the long-dashed-dotted curve, as expected.

To obtain a KS-reconstruction where almost no information on data outside $\mathcal{U}$ is used, we increase the noise outside $\mathcal{U}$ by doubling the width of the ellipticity distribution for galaxies outside $\mathcal{U}$. The shear field is calculated in the large field and the surface density is KS-reconstructed. The power spectrum of the reconstruction error within the small field is shown as short-dashed-dotted line - and it is very similar to the power spectrum of the finite field NFreconstruction. One could argue that this large increase is caused mainly by the fact that by the averaging procedure (8) the increased noise outside $\mathcal{U}$ is partly tranferred in $\mathcal{U}$. To show that this is not true we again consider the case where the shear field is calculated in the large region as before, but where the values inside $\mathcal{U}$ are the same as used for the KS- and NF-reconstruction of the small field $\mathcal{U}$. We find that the reconstruction error is then only marginally decreased (short-dashed curve). But still one could argue that in this case the possibly non-smooth transition from the $g$-field inside $\mathcal{U}$ to that outside could significantly contribute to the noise. Therefore we smoothed that transition on the neighboring gridpoints outside the data field. The corresponding power spectrum (long-dashed curve) shows that the smootheness of this transition has only a small effect on the noise properties of the reconstruction within $\mathcal{U}$. This comparison demonstrates that the KSreconstruction becomes worse the noisier the data outside $\mathcal{U}$ are and that the assumption of $\gamma \equiv 0$ outside $\mathcal{U}$ is responsible for the high quality of the KS-reconstruction if there is no mass in the field. Since this is not the case for most fields currently observed, one is urged to use a method which is exact on finite fields (see Squires \& Kaiser 1996).

Finally we apply the new noise filtering to the WFPC-2 geometry. Instead of performing a power-spectrum analysis, we have calculated the mean-square deviation of the

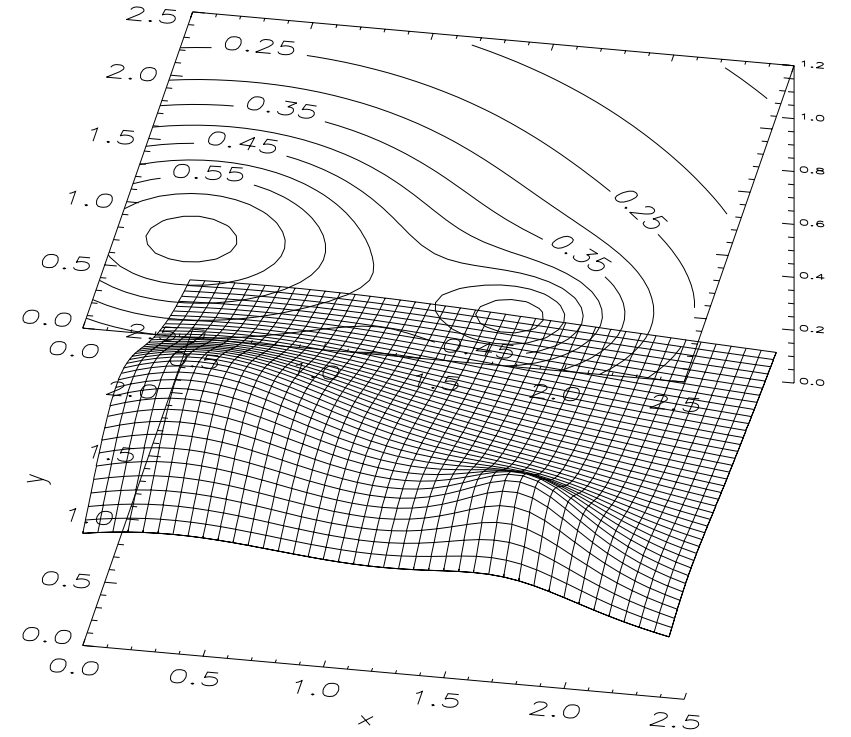

Fig. 2. This mass distribution was used when the rms-error for the NF and NF1 are compared in Fig. 3; it was chosen to similar to that of the cluster Cl0939. The contours and surface plot shows $-K(\boldsymbol{x})=-\ln [1-\kappa(\boldsymbol{x})]$. The grid is $40 \times 40$ and the field of view is 2.5 arcmin on a side.

reconstructed density field $K(\boldsymbol{\theta})$ (shifted such that the mean value of $K$ over the field $\mathcal{U}$ equals the true one) from the input distribution (see also Fig. 10 in Paper I). We consider again two cases, the "no-lens-case" and that of a mass distribution which was now chosen similar to that in the cluster $\mathrm{Cl} 0939$ (see Fig. 2) For both cases the galaxy density (60 per square arcminute), the width of the ellipticity distribution $(\rho=0.2)$ and the smoothing length ( $\Delta \theta=0$.3 3 ) were chosen equal to the values for the weak lensing reconstruction of the cluster Cl0939 (Seitz et al. 1996). The reconstructions were obtained on a $40 \times 40$ grid. In each of the two cases, two different reconstructions are analyzed, one where the reconstruction was performed on a square with 2.5 sidelength, and the other where one quarter of the square was removed. Figure 3 shows the rms deviation for these cases, obtained from 500 realizations for each case. For illustration, only the WFPC-2 part of the square is shown in the first case. When compared to reconstructions on the square, the WFPC-2 reconstructions are just slightly more noisy close to the additional boundaries of the field, owing to the smaller number of galaxies from which the shear is obtained there. Note that the increase of noise at the "inner corner" of the WFPC-2 is much smaller than that at the "outer corners", which is due to the fact that at the former, more galaxies fall into the filter scale than in the latter case. The increase of the noise at those positions where the mass distribution peaks is due to the lack of spatial resolution of the inversion, due to the smoothing applied. In contrast to Paper I we have not attempted here to adopt an adaptive smoothing, depending on the lens signal, which would yield better resolution near the mass peaks. 

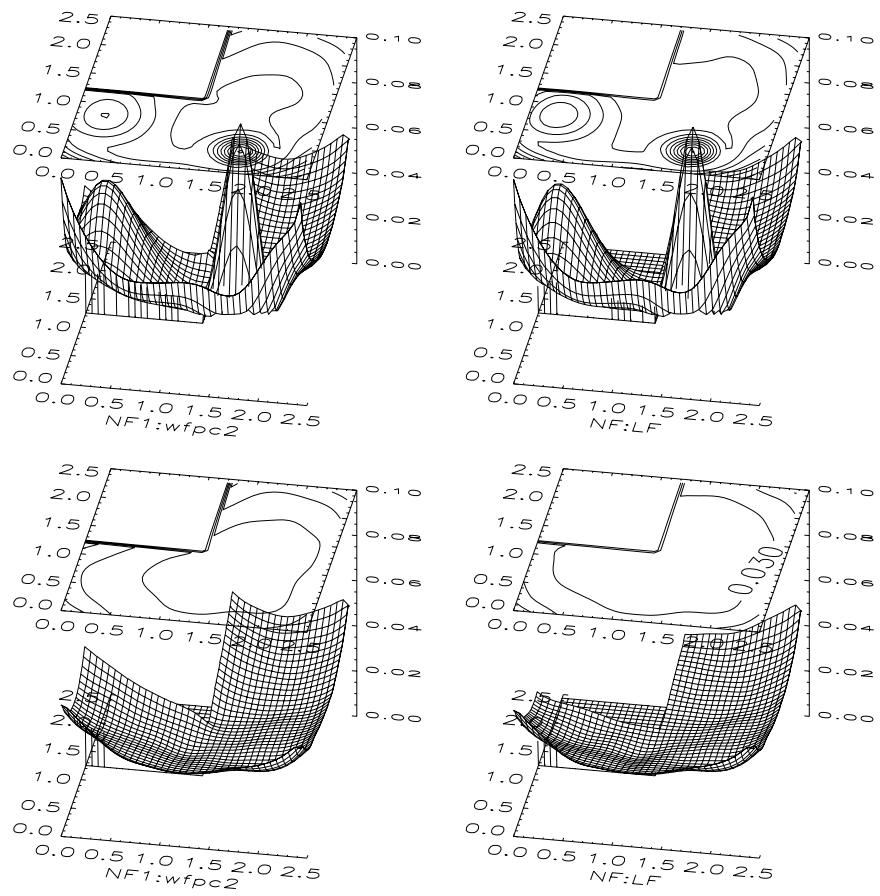

Fig. 3. The mean rms deviation (defined as $\Delta K$ p. 396 in Paper I) obtained from 500 reconstructions. The two left panels show $\Delta K$ obtained from NF1-reconstructions on the WFPC-2 shaped field, for the right panels we performed the NF-reconstructions on the quadratic 2.'5-field, but only show $\Delta K$ on the WFPC-2 field. In the upper panel, we used the mass model shown in Fig. 2, wheras in the the lower panels no mass was assumed.

\section{Conclusions}

We have derived a new version of the noise-filtering cluster mass reconstruction algorithm originally proposed in Paper I, which is easier to implement, easier to use on large fields where the required number of gridpoints can quickly exceed the number possible in using the method of Paper I, and which can easily be generalized to more complicated geometries; the particularly relevant case of the WFPC-2 geometry was considered explicitly. From extensive numerical tests we have shown that the noise properties of this version is basically identical to that of the method described in Paper I. In agreement with Fig. 6 of Squires \& Kaiser (1996), we conclude that the noise-filtering method is the best known direct finite-field inversion method. The comparison between the maximum probability method (Squires \& Kaiser 1996) and the method presented here, carried out on the mosaic of WFPC-2 centered on the cluster MS1358+62 (Hoekstra et al. 1998), yielded no easily visible difference in performance of these two methods.

Acknowledgements. We thank Bill Press for a very fruitful discussion which triggered the work presented here. This work was supported by the "Sonderforschungsbereich 375-95 für AstroTeilchenphysik" der Deutschen Forschungsgemeinschaft.

\section{References}

Bartelmann, M. 1995, A\&A, 303, 643

Bartelmann, M., \& Narayan, R. 1995, ApJ, 451, 60

Bartelmann, M., Narayan, R., Seitz, S., \& Schneider, P. 1996, ApJL, 464, 115

Broadhurst, T. J., Taylor, A. N., \& Peacock, J. A. 1995, ApJ, 438, 49

Gorenstein, M. V., Falco, E. E., \& Shapiro, I. I. 1988, ApJ, 327,693

Hoekstra, H., Franx, M., Kuijken, K., \& Squires, G. 1998, ApJ, 504,636

Kaiser, N. 1995, ApJ, 493, L1

Kaiser, N., \& Squires, G. 1993, ApJ, 404, 441 (KS)

Kaiser, N., Squires, G., \& Broadhurs, T. 1995, ApJ, 449, 460

Lombardi, M., \& Bertin, G. 1999, A\&A, 348, 38

Press, W. H., Teukolsky, S. A., Vettering, W. T., \& Flannery, B. P. 1992, Numerical Recipes (Cambridge University Press)

Schneider, P. 1995, A\&A, 302, 639

Schneider, P., \& Seitz, C. 1995, A\&A, 294, 411

Seitz, C., \& Schneider, P. 1995, A\&A, 297, 287

Seitz, C., \& Schneider, P. 1997, A\&A, 318, 617

Seitz, C., Kneib, J. P., Schneider, P., \& Seitz, S. 1996, A\&A, 314,707

Seitz, S., \& Schneider, P. 1996, A\&A, 305, 383 (Paper I)

Seitz, S., Schneider, P., \& Bartelmann, M. 1998, A\&A, 337, 325

Squires, G., \& Kaiser, N. 1996, ApJ, 473, 65

Tyson, J. A., Valdes, F., \& Wenk, R. A. 1990, ApJ, 349, L1 